\title{
Primer reporte en Chile de Chrysomya albiceps (Diptera: Calliphoridae) en evidencia entomológica forense
}

\author{
First report in Chile of Chrysomya albiceps (Diptera: Calliphoridae) \\ in forensic entomological evidence
}

\author{
A Ortloff-Trautmanna*, A Jara-Peñailillob, S Albornoz-Muñoz ${ }^{\mathrm{a}}$, R Silva-Riveros ${ }^{\mathrm{c}}$, \\ MP Riquelme-Gatica ${ }^{a}$, P Peña-Rehbeina \\ aEscuela de Medicina Veterinaria, Facultad de Recursos Naturales, \\ Universidad Católica de Temuco, Temuco, Chile. \\ bDepartamento de Criminalística de Carabineros de Chile (LABOCAR), Santiago, Chile. \\ cInstituto de Histología, Patología y Anatomía, Facultad de Medicina, \\ Universidad Austral de Chile, Valdivia, Chile.
}

\begin{abstract}
SUMMARY
Chrysomya albiceps (Wiedemann) (Diptera: Calliphoridae) is a hemisynanthropic fly widely distributed in Latin America. This organism is probably the most important fly species for forensic entomology in human and veterinary casuistic. Until now, there is no evidence of the presence of this species in Chile. We analysed entomological evidence collected from 60 human bodies found by Carabineros de Chile (Labocar) from 2006 through

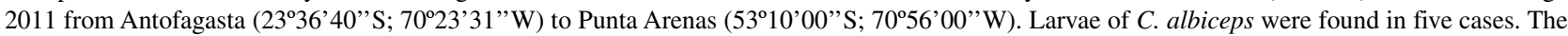

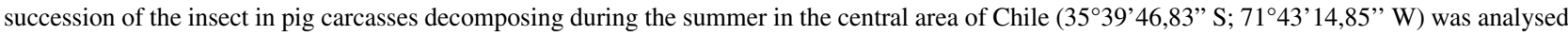
to report experimental evidence of the presence of $C$. albiceps in Chile and to record its life cycle. The development time of the fly from egg to imago emergence was 12 days. The largest $10 \%$ of the larvae in every sample was measured and data was presented in a table that can be used in Chilean forensic entomology. This is the first report of the presence of $C$. albiceps in Chile in forensic cases and we provide the first table containing the life cycle of this species in pig carcasses, this information could be used in forensic analysis for human and veterinary entomology.
\end{abstract}

Palabras clave: Chrysomya albiceps, Chile, entomología forense.

Key words: Chrysomya albiceps, Chile, forensic entomology.

\section{INTRODUCCIÓN}

Entomología forense (EF) es el nombre que recibe el estudio de los insectos que forman parte de la evidencia en casos legales, especialmente en sucesos de muerte (Amendt y col 2007). La aplicación más común de esta disciplina científica es la estimación de la data de muerte o intervalo post mortem mínimo (IPM ${ }_{\min }$ ) (Catts 1992); sin embargo, las diferentes técnicas que se han implementado en EF han permitido en ciertos casos proveer información concerniente a posible movimiento o almacenaje de cuerpos, intervalo de sumersión, tiempo de decapitación y/o desmembramiento de un cuerpo, identificación de sitios específicos de trauma, demostración de uso de drogas, culpar o exculpar a un sospechoso con la escena del crimen y demostrar que personas y/o animales han sufrido de negligencia o abandono durante un período de tiempo mediante el examen de los insectos en lesiones o miasis (Amendt y col 2011). El tipo de miasis más importante

Aceptado: 04.10.2012.

* Manuel Montt 056, Temuco, Chile; aortloff@uct.cl desde el punto de vista de la EF es la provocada por moscas de la familia Calliphoridae, los cuales pueden ser asociados a casos de negligencia extrema. En el caso veterinario, el rol del entomólogo forense es determinar el período de infestación del ganado o mascota en casos de crueldad o negligencia médica (Amendt y col 2011). Las especies de Calliphoridae más frecuentemente identificadas en miasis en humanos y animales son Cochliomyia homnivorax (Correia y col 2010), Lucilia sericata (Reyes y col 1967, Amendt y col 2011, Sotiraki y Hall 2012) y Chrysomya albiceps (Richard y col 2008, Sotiraki y Hall, 2012). Estas dos últimas especies son parásitos facultativos, por lo cual tienen gran importancia desde el punto de vista médico y forense (Anderson y Huitson 2004, Amendt y col 2011).

Chrysomya albiceps (Wiedemann) (Diptera: Calliphoridae) es una especie hemisinantrópica, que actúa como vector mecánico de enteropatógenos, causa miasis facultativa en humanos y animales domésticos (Hall y Smith 1993), y se alimenta y reproduce prolíficamente en cadáveres en descomposición y heces fecales, por lo que esta especie es importante desde el punto de vista médico, sanitario, veterinario y forense (Richard y col 2008). Se estima que $C$. albiceps es originaria de África y que ingresó 
a América del Sur en Brasil (Guimaraes y col 1978). Varios autores han observado que su introducción al Nuevo Mundo ha tenido un fuerte impacto en la abundancia de dípteros endémicos. Es probable que este impacto se deba a la capacidad depredadora facultativa y al comportamiento agresivo de esta especie durante su etapa larvaria, pudiendo alterar la composición de especies de dípteros que se desarrollan en un cadáver, lo cual tiene gran relevancia en el análisis entomológico forense (Faria y col 1999).

La presencia de C. albiceps en América Latina ha sido reportada en Argentina, Bolivia, Brasil, Ecuador, Venezuela, Colombia, Puerto Rico y Perú. En el catálogo de Calliphoridae de Chile (2006) se menciona que esta especie estaría en Chile, sin embargo no existen reportes desde el punto de vista entomológico forense en nuestro país. En Chile existe la investigación sobre mesofauna asociada a cadáveres de conejo en Valparaíso (Saiz y col 1989), sobre Calliphoridae realizada en Valdivia (Figueroa-Roa y Linhares 2002) y en Temuco con cadáveres de cerdo (Ortloff y col 2012). Ninguna de las tres investigaciones reportó la presencia de esta especie. Figueroa-Roa y Linhares (2002) explican que la ausencia de $C$. albiceps en esa región se debe a la baja temperatura media que caracteriza esta zona y esta especie no está adaptada a bajas temperaturas. No hay información sobre esta mosca en otras regiones de Chile.

La EF ha recibido poca atención en Chile a pesar del considerable valor que se le ha dado a esta disciplina en varios países de Latinoamérica como Argentina (Oliva 1997, Centeno y col 2002, Battán Horenstein y col 2010), Colombia (Wolff y col 2001, Segura y col 2009), Brasil (Carvalho y Linhares 2001, Gomes y col 2009) y Perú (Iannacone 2003). Debido a que C. albiceps es una de las especies de mosca más frecuentemente encontradas en cadáveres humanos y de animales, probablemente es una de las especies de díptero más importante en EF (OliveiraCosta y col 2001, Grassberger y col 2003, Carvalho y col 2004, Gomes y col 2009).

Debido a la amplia distribución de C. albiceps en los países cercanos a Chile y la importancia de ésta en las investigaciones forenses humanas y veterinarias, así como su implicancia ecológica y médica, se decidió investigar el estatus de esta especie en Chile. Esta es la primera publicación que presenta evidencia sobre la presencia de esta especie de importancia forense humana y veterinaria en muestras entomológicas forenses en Chile, así como también se presenta la primera tabla con parámetros larvarios que podrá utilizarse en pericia entomológica forense en Chile.

\section{MATERIAL Y MÉTODOS}

\section{CASUÍSTICA DE ENTOMOLOGÍA FORENSE EN CHILE}

Para encontrar evidencia sobre la presencia de Chrysomya albiceps en cadáveres humanos en Chile y determinar aproximadamente su distribución a lo largo del país, se analizó la evidencia entomológica recolectada por el Laboratorio de Criminalística de Carabineros de Chile en todos los cadáveres humanos periciados desde el año 2006 al año 2011. La colección consta de 60 casos de cadáveres humanos encontrados en primavera y verano desde Antofagasta (2336'40's 70²3'31'O) hasta Punta

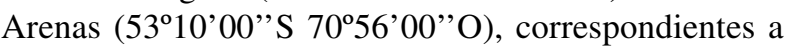
casos que demandaron pericia entomológica. Todas las muestras recolectadas correspondían a larvas, pupas y/o adultos de dípteros que fueron identificadas utilizando claves taxonómicas (Smith 1986, Florez y Wolff 2009).

\section{ESTUDIO EXPERIMENTAL}

Una vez determinada la presencia de $C$. albiceps mediante el análisis de la evidencia recolectada en la casuística, se diseñó el siguiente experimento para elaborar una tabla con parámetros larvarios utilizable en pericia entomológica forense:

Localización geográfica y estación del año: El experimento se realizó en febrero del año 2011 en un área rural de Villa Alegre, Región del Maule (35³9'46”S, 7143'14”O), a 108 metros sobre el nivel del mar.

Material biológico: Se utilizaron cuatro cerdos (Sus scrofa Linnaeus) de $20 \mathrm{~kg}$, los cuales fueron sacrificados en el lugar mediante trauma encefalocraneal. Este procedimiento fue aprobado por el Comité de Ética de la Universidad Católica de Temuco. Los cadáveres fueron ubicados a 20 metros de distancia entre sí y protegidos por jaulas de malla metálica de $100 \mathrm{~cm}$ x $80 \mathrm{~cm}$ x $60 \mathrm{~cm}$ para evitar la depredación por carroñeros vertebrados, pero sin alterar el acceso a entomofauna cadavérica.

Registros diarios: Se inspeccionaron los cadáveres todos los días entre las 15:00 y 17:00 h, se tomaron fotografías y se determinó el estado del proceso de descomposición según Anderson y Vanlaerhoven (1996). Simultáneamente se registró i) la temperatura de los cadáveres vía rectal con termómetro digital, ii) la temperatura de las masas larvarias con termómetro digital y iii) la temperatura ambiental máxima y mínima del día mediante termómetro de máxima y mínima.

Muestras biológicas: Uno de los cadáveres no fue muestreado (control) para corroborar al final del experimento que la intervención de los investigadores en los cadáveres no alteró el proceso de descomposición. i) Se capturaron insectos voladores y caminadores utilizando red entomológica y pinzas de acuerdo a Haskell y col (2001) y se preservaron en etanol al 80\%. ii) En cada cadáver se identificaron diariamente los nuevos racimos de huevos de dípteros y se tomó una pequeña muestra (aproximadamente 15 huevos por racimo), los cuales se criaron en sustrato alimenticio (hígado de bovino) dentro de envases con arena hasta obtener insectos adultos. De esta forma se identificó a las 
especies colonizadoras primarias del cadáver. iii) De cada cadáver se tomaron diariamente muestras representativas de las masas larvarias. Para esto se dividió el cadáver en dos regiones topográficas: anterior (que incluye cabeza, cuello y miembros anteriores) y posterior (que incluye tórax, abdomen y extremidades posteriores). De cada región se tomaron cinco muestras de masas larvarias utilizando pinzas, pincel y/o cuchara (dependiendo del tamaño de las larvas) y se fijaron de acuerdo a Adams y Hall (2009).

Análisis de las larvas: La medición de la longitud de la larva más grande de una muestra (es decir la de mayor edad, excepto en las larvas post-alimentarias y prepupas) es una práctica común en EF para determinar $\mathrm{IPM}_{\text {min }}$ (Grassberger y Reiter 2001), por ello se seleccionó el 10\% de las larvas de mayor tamaño de cada muestra y se midieron utilizando un estereomicroscopio Olympus SZ61 con objetivo graduado en $0,2 \mathrm{~mm}$. Posteriormente se determinó el estado de desarrollo de todas las larvas (instar) y la especie. Cuando se observó la migración de larvas post-alimentarias en el suelo, se comenzó a analizar diariamente el suelo para encontrar pupas. Como en la metamorfosis de un insecto el estado de pupa es más avanzado (de más edad) que el estado de larva, encontrar pupas en un muestreo entomológico forense entrega más información para calcular el IPM $_{\text {min }}$ que las larvas, por lo que la medición de la longitud de las larvas en el experimento finalizó cuando se encontraron las primeras pupas en el suelo.

Claves taxonómicas: Para identificar Calliphoridae adultos se utilizó la clave de Amat (2009) y para las larvas, la clave de Florez y Wolff (2009), además de la descripción morfológica de Carvalho y col (1997).

Microscopía electrónica de barrido (MEB): Una vez identificadas las larvas del género Chrysomya, se utilizó MEB para diferenciar $C$. albiceps de $C$. rufifacies mediante la observación de las espinas apicales de los tubérculos. Se utilizó el protocolo descrito por Mendonça y col (2010) y se comparó con las descripciones morfológicas ultraestructurales de estos autores y de Wells y col (1999).

Todas las observaciones taxonómicas tanto de larvas como de los adultos recolectados fueron corroboradas por el entomólogo forense Dr. Néstor Centeno (perito del Ministerio Público Fiscal y académico de la Universidad Nacional de Quilmes, Buenos Aires-Argentina).

\section{RESULTADOS Y DISCUSIÓN}

\section{PRINCIPALES CRITERIOS TAXONÓMICOS PARA LA IDENTIFICACIÓN DE LOS ADULTOS}

- Base de la vena radial (R) (vena troncal o vena remigio) ventralmente desnuda: subfamilia Chrysomyinae.

- Ampolla cubierta con pilosidad larga; calíptero inferior dorsalmente piloso en toda la superficie y subtruncado

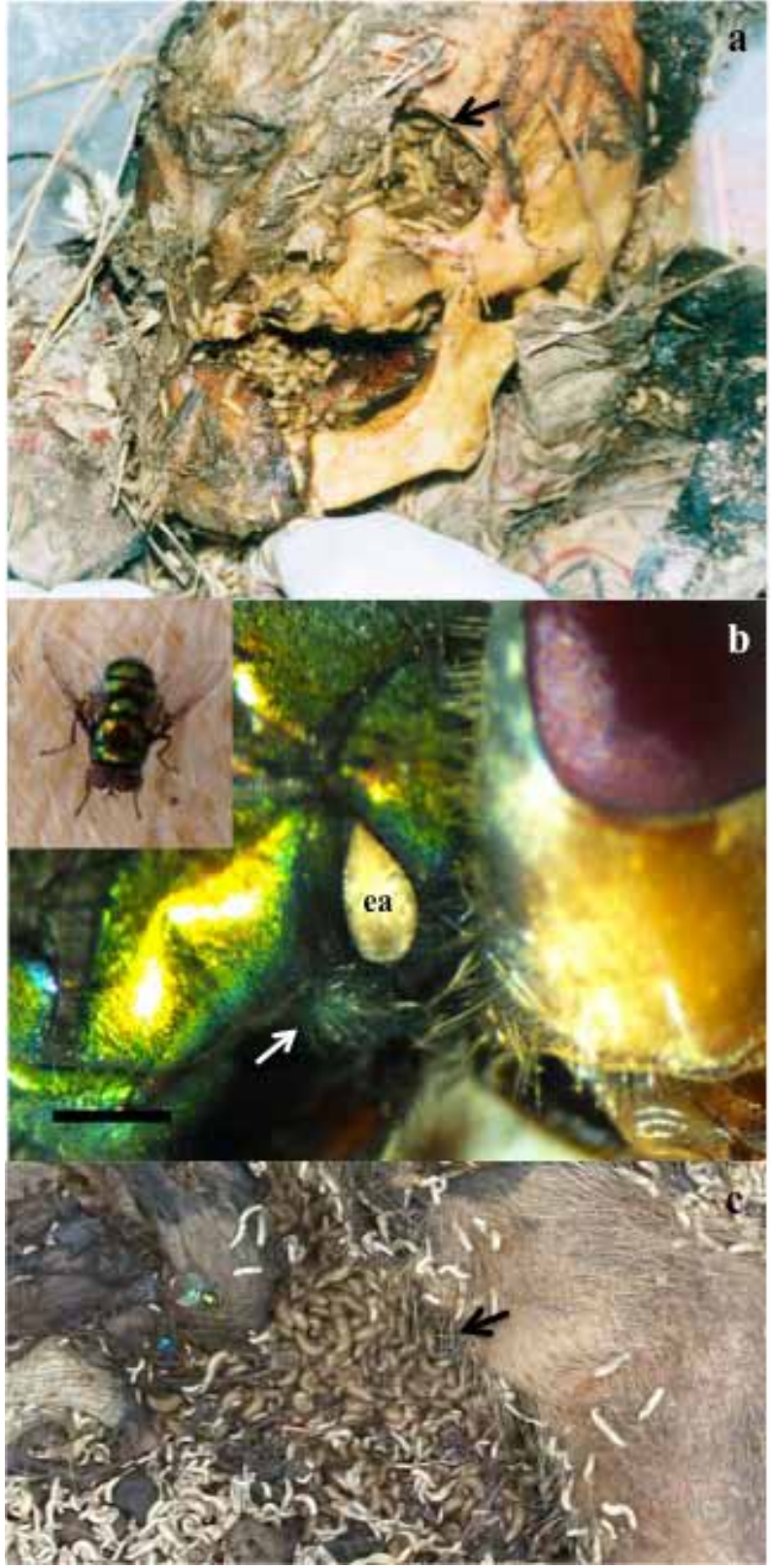

Figura 1. a. Caso 1, cadáver en estado de restos. Larvas instar III de C. albiceps in cavidad orbitaria (flecha negra) y boca. b. Detalle de tórax de imago de $C$. albiceps, vista lateral. Ausencia de seta estigmática (flecha blanca). En $C$. rufifacies esta seta está presente. ea: espiráculo anterior. Escala: $500 \mu \mathrm{m}$. Inserto: Adulto de $C$. albiceps sobre cadáver de cerdo. c. Masa larvaria de $C$. albiceps (flecha negra) en cadáver de cerdo.

a. Case 1, man in remains stage. Note larvae instar III of $C$. albiceps in eye socket (black arrow) and mouth. b. Detail of the thorax of C. albiceps imago, side view. Absence of stigmatic setae (white arrow). In C. rufifacies this setae is present. ea: anterior spiracle. Scale: $500 \mu \mathrm{m}$. Insert: Adult C. albiceps on pig carcass. c. Larval masses of $C$. albiceps (black arrow) in pig carcass.

posteriormente con el borde externo angular: Chrysomya Robineau-Desvoidy, 1830.

- Cerda estigmática ausente y 4-6 cerdas proepisternales presentes (figura 1b): C. albiceps (Wiedemann 1819). 
PRINCIPALES CRITERIOS TAXONÓMICOS PARA LA

IDENTIFICACIÓN DE LAS LARVAS INSTAR III

- Segmentos 3-12 con hileras de tubérculos conspicuos dorsalmente, segmentos 3 y 4 con tubérculos más pequeños y tubérculos ventrales de menor tamaño (figura 2a).

- Tubérculos dorsales con espinas apicales relativamente pequeñas, usualmente apuntando hacia el centro (figura 2d,e) .

- Cuernos dorsal y ventral de igual longitud.

- Espiráculos anteriores con 8-12 branquias (figura 2b,c).

- Espiráculos posteriores con peritrema abierto (incompleto) muy pigmentado y botón imperceptible (figura $2 \mathrm{f}$ ).

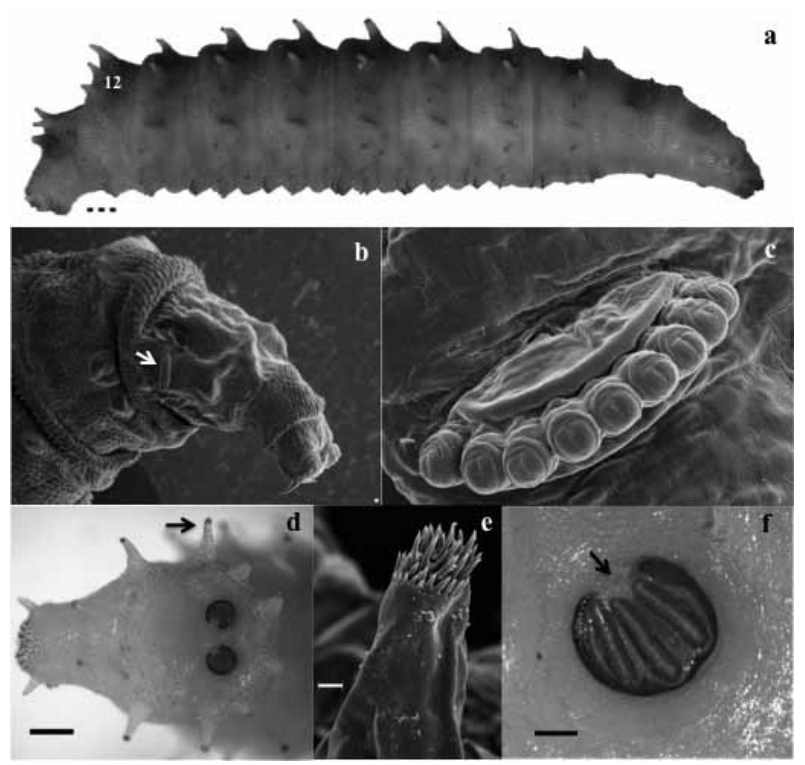

Figura 2. a. Larva instar III de C. albiceps. 12: segmento 12. Escala: $500 \mu \mathrm{m}$. b. Detalle ultraestructural de extremo anterior de larva instar III de $C$. albiceps colectada de un cadáver de cerdo. Flecha blanca: espiráculo anterior. c. Detalle ultraestructural de espiráculo anterior de larva instar III. Esta larva tiene 9 branquias. d. Extremo posterior de larva instar III. Flecha negra: tubérculos laterales. Escala: $400 \mu \mathrm{m}$. e. Detalle ultraestructural de espinas apicales en tubérculo lateral en una larva instar III de d. Escala: $20 \mu \mathrm{m}$. f. Detalle de espiráculo posterior de la larva en d. Flecha negra: botón imperceptible y peritrema abierto. Escala: $100 \mu \mathrm{m}$.

a. Larvae instar III of $C$. albiceps. 12: segment 12. Scale: $500 \mu \mathrm{m}$. b. Ultrastructural detail of anterior end of larval instar III of C. albiceps collected from a pig carcass. White arrow: anterior spiracle. c. Ultrastructural detail of anterior spiracle of larvae instar III. This larvae has 9 gills. d. Back end of larvae instar III. Black arrow: lateral tubercles. Scale: $400 \mu \mathrm{m}$. e. Ultrastructural detail of apical spines on the lateral tubercle of larvae instar III of d. Scale: $20 \mu \mathrm{m}$. f. Detail of posterior spiracle of larvae in d. Black arrow: imperceptible button and opened peritrema. Scale: $100 \mu \mathrm{m}$.
CHRYSOMYA ALBICEPS EN CASUÍSTICA FORENSE HUMANA EN CHILE

De los 60 casos de cadáveres humanos muestreados para análisis entomológico forense, se encontró en cinco de ellos la presencia de larvas y pupas de C. albiceps; los cadáveres presentaban las siguientes características:

- Caso 1: Sexo masculino en estado esquelético (figura 1a). Encontrado en ambiente externo en un área semirrural en Las Vizcachas, Región Metropolitana (33³4'32''S 70³3'06”'O).

- Caso 2: Sexo femenino en estado enfisematoso. Encontrado en ambiente externo en un área rural cerca de Buin, Región Metropolitana (3345'60'S 70³9'39'O).

- Caso 3: Sexo masculino en estado enfisematoso. Encontrado en ambiente externo en un área semirrural cerca de San Antonio, Región de Valparaíso (33³7’25”S $\left.71^{\circ} 35^{\prime} 58^{\prime \prime} \mathrm{O}\right)$.

- Caso 4: Sexo masculino en estado de descomposición avanzada. Encontrado en el interior de una casa en un área rural de Pirque, Región Metropolitana (33³6'09's $\left.70^{\circ} 29^{\prime} 48^{\prime \prime} \mathrm{O}\right)$.

- Caso 5: Sexo masculino en estado de descomposición activa. Encontrado en ambiente externo en un área rural cerca de Rancagua, Región del Libertador General Bernardo O'Higgins (3403'49' S 7040'36”O).

\section{ESTUDIO EXPERIMENTAL USANDO CADÁVERES DE CERDO}

Todos los registros obtenidos durante el experimento se muestran en la tabla 1. Los tres cadáveres mostraron el mismo patrón y duración de los estados de descomposición que el cadáver control. Se reconocieron cinco estados: Fresco, Enfisematoso o hinchado, Descomposición activa, Descomposición Avanzada y Restos esqueléticos. El proceso de descomposición duró 14 días desde el estado Fresco al estado Esquelético (cuadro 1).

Los días 1, 2 y 3 se caracterizaron por la llegada de dípteros adultos de las especies Chrysomya albiceps, Lucilia sericata, Cochliomyia macellaria (Diptera: Calliphoridae) y Musca domestica (Diptera: Muscidae). En estos días hubo postura de huevos y desarrollo de las primeras masas larvarias. De acuerdo a la crianza de los huevos y observación de las larvas, estas primeras masas larvarias correspondieron a las especies $C$. albiceps, L. sericata y C. macellaria, por lo cual fueron consideradas especies colonizadoras de cadáveres en esta zona de Chile. Estas observaciones son similares a lo reportado en Argentina (Centeno y col 2002, Battán Horenstein y col 2010) y Brasil (Gomes y col 2009). La identidad de las larvas de $C$. albiceps fue corroborada en el análisis de MEB (figura 2).

Los estados de descomposición activa y avanzada se caracterizaron por la dominancia de larvas de $C$. albiceps 


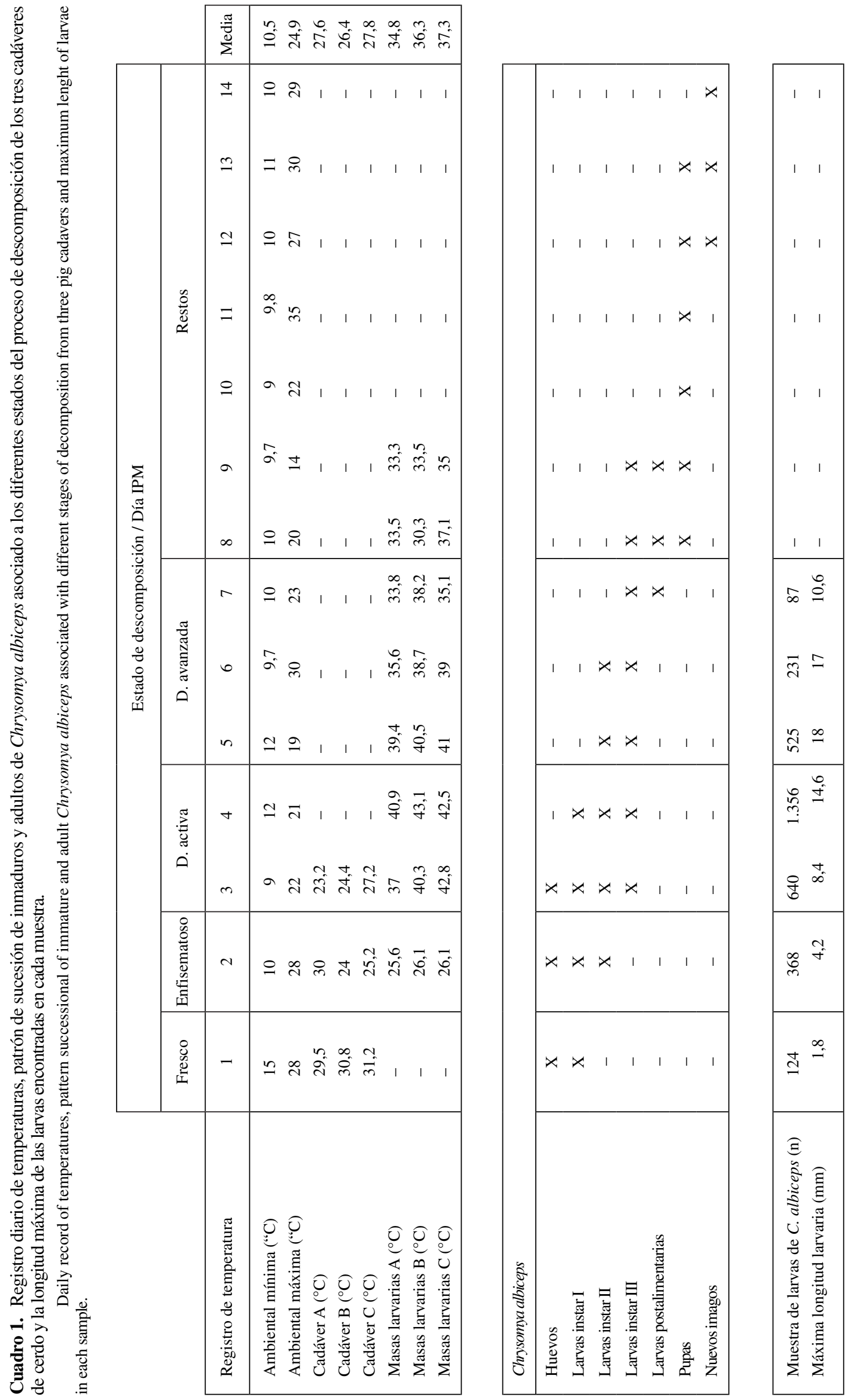


sobre las larvas de las otras especies, observándose larvas de $C$. albiceps instar III depredando otras larvas. Desde el día quinto en adelante las larvas de L. sericata y C. macellaria aparecieron en escaso número en las muestras de masas larvarias. Esta situación también fue observada en Argentina en cadáveres de cerdo, en los cuales larvas de C. albiceps depredaron larvas de otras especies, quedando finalmente sólo larvas de $C$. albiceps sobre el cadáver (Centeno y col 2002). El comportamiento depredador facultativo de las larvas de $C$. albiceps ha demostrado tener un efecto negativo sobre especies de dípteros nativos como Cochliomyia macellaria (Faria y col 1999). Este es el primer reporte en Chile del efecto que tienen las larvas C. albiceps sobre las otras especies necrobiontes en cadáveres, sin embargo se desconoce el impacto ecológico de este fenómeno.

Desde el punto de vista forense, la eliminación de las larvas de las otras especies colonizadoras tempranas de cadáveres producto de la depredación tiene consecuencias importantes al momento de querer calcular el IPM $_{\text {min }}$ basándose en el análisis de éstas, ya que se está poniendo el reloj post mórtem de insectos erróneamente "en cero" al no encontrar larvas de las especies descritas como colonizadoras primarias (Grassberger y col 2003). Es por esto que en las zonas geográficas donde $C$. albiceps está presente se utiliza esta especie como principal bioindicador de IPM ${ }_{\text {min }}$ (Grassberger y col 2003).

El patrón de distribución preliminar de $C$. albiceps en Chile obtenido a partir de los registros periciales podría ser utilizado en casos policiales donde se sospeche que un cadáver fue movilizado de una región a otra. Por ejemplo, si se encuentra un cadáver en Valdivia con larvas de C. albiceps, es posible que el sitio del suceso de muerte haya sido en una zona geoclimática donde está presente esta especie y luego fue movilizado hacia Valdivia donde C. albiceps normalmente no se encuentra.

En el ambiente experimental el tiempo de desarrollo de esta especie fue de 8 días desde huevo a pupa, y de 12 días de huevo a la emergencia de los adultos (tabla 1). Estos valores son comparables con los observados por Marchenko (2001) y Grassberger y col (2003) en condiciones in vitro a temperatura constante de $25^{\circ} \mathrm{C}$. El promedio de temperatura ambiente obtenido en el presente estudio fue de $17,7^{\circ} \mathrm{C}$, por lo que es posible que la temperatura de las masas larvarias $\left(36,1{ }^{\circ} \mathrm{C}\right.$ en promedio en este experimento) mantuvo un ambiente cálido que favoreció este rápido desarrollo (Charabidze y col 2011).

En conclusión, la evidencia pericial y experimental confirman que $C$. albiceps está presente en Chile al menos

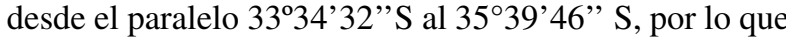
podría utilizarse como bioindicador en pericia entomológica forense chilena, tanto como para aproximar un cálculo del IPM mínimo, guiar investigaciones sobre movimiento de cadáveres, demostrar negligencia médica humana y/o veterinaria, entre otros usos.

\section{RESUMEN}

Chrysomya albiceps (Wiedemann) (Diptera: Calliphoridae) es una mosca hemisinantrópica ampliamente distribuida en América Latina. Es probable que esta especie de mosca sea la más importante para la entomología forense en casuística humana y veterinaria. Hasta la fecha, no hay evidencia de la presencia de esta especie en Chile. En esta investigación se analizó la evidencia entomológica recogida de 60 cadáveres humanos encontrados por Carabineros de Chile (Labocar) desde el año 2006 hasta el 2011 desde Antofagasta (2336' $40^{\prime \prime}$ S, 70²3'31",

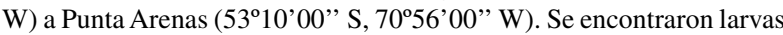
de $C$. albiceps en cinco casos. Para ofrecer la evidencia experimental de la presencia de $C$. albiceps en Chile y para generar una tabla con su ciclo de vida, se analizó la sucesión de insectos en cadáveres de cerdo en descomposición durante el verano en la zona central de Chile (3539'46,8'S, $\left.71^{\circ} 43^{\prime} 14,85^{\prime \prime} \mathrm{O}\right)$. El tiempo de desarrollo de la mosca de huevo a imago fue de 12 días. El 10\% de las larvas más grandes de cada muestra se midió para desarrollar una tabla que podrá ser utilizada en análisis entomológico forense en Chile. Este es el primer reporte de la presencia de $C$. albiceps en Chile en casos forenses y se muestra la primera tabla con el ciclo de esta especie en cadáveres de cerdo que puede ser utilizado en el análisis entomológico forense humano y veterinario.

\section{AGRADECIMIENTOS}

Los autores agradecen la asesoría del entomólogo forense Dr. Néstor Centeno, Perito del Ministerio Público Fiscal y académico de la Universidad Nacional de Quilmes, Buenos Aires-Argentina.

\section{REFERENCIAS}

Adams ZJO, MJR Hall. 2003. Methods used for the killing and preservation of blowfly larvae, and their effect on post-mortem larval lenght. Forensic Sci Int 138, 50-61.

Amat E. 2009. Contribución al conocimiento de las Chrysomyinae y Toxotarsinae (Diptera: Calliphoridae) de Colombia. Revista Mexicana de Biodiversidad 80, 693-708.

Amendt J, CP Campobasso, E Gaudry, C Reiter, HN LeBlanc, MJR Hall. 2007. Best practice in forensic entomology - standards and guidelines. Int J Legal Med 121, 90-104.

Amendt J, CS Richards, CP Campobasso, R Zehner, MJR Hall. 2011. Forensic entomology: applications and limitations. Forensic Sci Med Pathol 7, 379-392.

Anderson GS, SL Vanlaerhoven. 1996. Initial studies on insect succession on carrion in Southwestern British Columbia. J Forensic Sci 42, 617-625.

Anderson GS, NR Huitson. 2004. Myiasis in pet animals in British Columbia: The potential of forensic entomology for determining duration of possible neglect. Can Vet J 45, 993-998.

Battán Horenstein M, AX Linhares, B Rosso de Ferradas, D García. 2010. Decomposition and dipteran succession in pig carrion in central Argentina: ecological aspects and their importance in forensic sciences. Med Vet Entomol 24, 16-25.

Carvalho MM, RP Mello, MM Lima. 1997. Morphological aspects of the larval instars of Chrysomya albiceps (Diptera, Calliphoridae) reared in the laboratory. Mem Inst Oswaldo Cruz 92, 187-196.

Carvalho LML, AX Linhares. 2001. Seasonality of insect succession and pig carcass decomposition in a natural forest area in southeastern Brazil. J Forensic Sci 46, 604-608.

Carvalho LML, PJ Thyssen, ML Goff, AX Linhares. 2004. Observations on the succession patterns of necrophagous insects onto a pig carcass in a urban area of Southeastern Brazil. Anal Aggrawal's Internet Journal of Forensic Medicine and Toxicology 5, 33-39.

Catts EP. 1992. Problems in estimating the post-mortem interval in death investigations. J Agric Entomol 9, 245-55.

Centeno N, M Maldonado, A Oliva. 2002. Seasonal patterns of arthropods occurring on sheltered and unsheltered pig carcasses in Buenos Aires Province (Argentina). Forensic Sci Int 126, 63-70. 
Charabidze D, B Bourel, D Gosset. 2011. Larval-mass effect: characterisation of heat emission by necrophageous blowflies (Diptera: Calliphoridae) larval aggregates. Forensic Sci Int 211, 61-66.

Correia TR, FB Scott, GG Verocai, CP Souza, JI Fernandes, RMPS Melo, VPC Vieira, FA Ribeiro. 2010. Larvicidal efficacy of nitenpyram on the treatment of myiasis caused by Cochliomyia homnivorax (Diptera: Calliphoridae) in dogs. Vet Parasitol 173, 169-172.

Faria LDB, L Orsi, LA Trinca, WAC Godoy. 1999. Larval predation by Chrysomya albiceps on Cochliomyia macellaria, Chrysomya megacephala, and Chrysomya putoria. Entomol Exp Appl 90, 149-155.

Figueroa-Roa L, AX Linhares. 2002. Sinantropia de los Calliphoridae (Diptera) de Valdivia, Chile. Neotrop Entomol 31, 233-239.

Florez E, M Wolff. 2009. Descripción y clave de los estadios inmaduros de las principales especies de Calliphoridae (Diptera) de importancia forense en Colombia. Neotrop Entomol 38, 418-429.

Gomes L, G Gomes, C Desuó. 2009. A preliminary study of insect fauna on pig carcasses located in sugar cane in Winter in southeastern Brazil. Med Vet Entomol 23, 155-159.

Grassberger M, C Reiter. 2001. Effect of temperatura on Lucilia sericata (Diptera: Calliphoridae) development with special reference to the isomegalen- and isomorphen-diagram. Forensic Sci Int 120, 32-36.

Grassberger M, E Friedrich, C Reiter. 2003. The blowfly Chrysomya albiceps (Wiedemann) (Diptera: Calliphoridae) as a new forensic indicator in Central Europe. Int J Legal Med 117, 75-81.

Guimaraes JH, AP Prado, AX Linhares. 1978. Three newly introduced blowfly species in Southern Brazil (Diptera, Calliphoridae). Rev Bras Entomol 22, 53-60.

Hall MJR, KGV Smith. 1993. Diptera causing myasis in man. In: Lane RP, Crosskey RW (eds). Medical insects and arachnids. Chapman and Hall, London, UK, Pp 429-469.

Haskell NH, WD Lord, JH Byrd. 2001. Collection of entomological evidence during death investigation. In: Byrd JH, Castner JL (eds). Forensic entomology - the utility of arthropods in legal investigations. CRC Press, Boca Raton, FL, USA, Pp 92-98.

Iannacone J. 2003. Artropofauna de importancia forense en un cadáver de cerdo en el Callao, Perú. Rev Bras Zoo 20, 85-90.

Mac-Lean M, CR González. 2006. Catálogo de los Calliphoridae de Chile (Diptera: Oestroidea). Acta Ent Chilena 30, 15-22.
Marchenko MI. 2001. Medicolegal relevance of cadaver entomofauna for the determination of the time of death. Forensic Sci Int 120, 89-109.

Mendonça PM, JR Dos Santos-Mallet, MM De Carvalho Queriroz. 2010. Ultramorphological characteristics on immature stages of Chrysomya albiceps (Wiedemann 1819) (Diptera: Calliphoridae), a fly specie of forensic importance. Microscopy Res Tech 73, 779-784.

Oliva A. 1997. Insectos de interés forense de Buenos Aires (Argentina). Primera lista ilustrada y datos bionómicos. Revista de Museo Argentino de Ciencias Naturales 'Bernardino Rivadavia' Entomología 7, 13-59.

Oliveira-Costa J, CA Mello-Patiu, SM Lopes. 2001. Muscoid diptera associated with human corpses at the death scene in the State of Rio de Janeiro, Brazil. Boletin do Museu Nacional, N.S. (Zool) 464, 1-6.

Ortloff A, P Peña, M Riquelme. 2012. Preliminary study of the succession pattern of necrobiont insects, colonising species and larvae on pig carcasses in Temuco (Chile) for forensic applications. Forensic Sci Int 222, e36-41.

Reyes H, H Hevia, H Schenone, J Sapunar. 1967. Human myiasis caused by Phaenicia sericata (Meigen, 1826) in Chile (Diptera: Calliphoridae). Bol Chil Parasitol 22, 168-171.

Richard CS, ID Paterson, MH Villet. 2008. Estimating the age of immature Chrysomya albiceps (Diptera: Calliphoridae), correcting for temperatura and geographical latitude. Int J Legal Med 122, 271-279.

Saiz F, E Tosti-Croce, M Leiva. 1989. Estudio de los cambios de la mesofauna asociada a la descomposición de cadáveres de conejo en clima mediterráneo. An Mus Hist Nat Valparaiso 20, 41-74.

Segura NA, W Usaquén, MC Sánchez, L Chuaire, F Bello. 2009. Succession pattern of cadaverous entomofauna in a semi-rural area of Bogotá, Colombia. Forensic Sci Int 187, 66-72.

Smith KGV. 1986. A manual of forensic entomology. British Museum (Natural History) and Cornell University Press, London, UK, Pp 102-120.

Sotiraki S, MJR Hall. 2012. A review of comparative aspects of myiasis in goats and sheep in Europe. Small Ruminant Research 103, 75-83.

Wells JD, JH Byrd, TI Tantawi. 1999. Key to third-instar Chrysomyinae (Diptera: Calliphoridae) from carrion in the continental United States. J Med Entomol 36, 638-641.

Wolff M, A Uribe, P Ortiz, A Duque. 2001. A preliminary study of forensic entomology in Medellín, Colombia. Forensic Sci Int 120, 53-59. 